\title{
Fabrication of Moth-eye Antireflective Nanostructures via Oxygen Ion-beam Etching on a UV-curable Polymer
}

\author{
Takao Okabe $^{1 *}$, Katsuyuki Yatagawa ${ }^{1}$, Kazuki Fujiwara ${ }^{1}$, and Jun Taniguchi ${ }^{1}$ \\ ${ }^{1}$ Department of Applied Electronics, Faculty of Advanced Engineering, \\ Tokyo University of Science, 6-3-1 Niijuku, Katsushika-ku, Tokyo 125-8585, \\ Japan \\ *okabe@te.noda.tus.ac.jp
}

\begin{abstract}
We report a low-cost, high-throughput process for fabricating a moth-eyelike anti-reflector on a curved surface. First, a curved surface was made from a UV-curable resin via UV imprinting. Then, the moth-eye-like structures were formed directly on the polymer surface via oxygen ion-beam etching. This produced flexible, transparent, and curved polymer products with anti-reflective properties. The method avoids duplication, which is generally needed for conventional anti-reflector fabrication. We compared the anti-reflective performance both flat and curved lenses before and after etching. The reflectance after etching for $150 \mathrm{~s}$ was substantially $10 \%$ lower than that of the non-etched surface. The usefulness of this method for polymer optical products with antireflective properties was demonstrated.
\end{abstract}

Keywords: Anti-reflection, Moth-eye, Nanostructures, UV nanoimprinting, Ionbeam etching

\section{Introduction}

Moth-eye-like structures are well known antireflectors (ARs), which have applications in devices such as solar cells [1], liquid crystal displays [2,3], and optical lenses [4-6]. Their nanostructure mimics that of a moth's eye, which has many nanoscale bumps on its surface, allowing it to gather light with high efficiency. To satisfy the increasing demand for AR devices, better methods to fabricate such nano-scale structures are required.

One of a most popular methods for moth-eye fabrication is a combination of etching (dry or wet) and nanoimprint lithography (NIL). In the etching process, moth-eye-like structures are formed on a hard material, such as silicon [2], metal [3,4], carbon [7-9], or a ceramic [10]. In this process, masking is sometimes used to make regularly arranged patterns. For example, Ji et al. reported a particle-masking method ('colloidal lithography') that uses a particle monolayer as a mask [11, 12]. Processes that do not need masking have also been reported, whereby the moth-eye-like nanostructures are etched on glassy carbon using an oxygen ion beam [7-9].

After etching, several NIL processes using a soft and flexible resin are needed to copy the etched moth-eye shape to the target devices or to additional NIL molds, as schematically shown in Fig. 1(a) [8, 13-15]. This is because it is difficult and costly to make smooth curved surfaces, such as lenses and roll-to-roll molds, directly on hard materials. Although these processes can be used to fabricate practical moth-eyes with low reflectance $[7-9,16$, 17], cheaper and higher-throughput methods are needed, particularly for AR applications in portable camera devices [18-20].

To meet the aforementioned demands, we propose a method to fabricate moth-eye-like nanostructures directly on a photo-curable polymer via oxygen ionbeam etching. Figure 1(b) shows the proposed method, which consist of two steps: UV imprinting and etching. First, a curved surface was fabricated 
using a UV-curable resin. The cured resin was then directly etched by the ion beam to form the motheye-like nanostructure. The use of a UV curable resin makes this process is both low-cost and highthroughput. Another advantage of the process is that there is no need for duplication of the structure onto a flexible and transparent polymer. This also lowers the cost and increases the throughput.

In this study, we formed moth-eye nanostructures on a UV-curable resin on flat and curved (concave and convex) surfaces and investigated their AR performance. In addition, AR etching on an UVNIL-duplicated lens array was demonstrated.

\section{Experimental}

\subsection{Materials}

The moth-eye-like structures were made using a UV-curable resin. The resin consisted of a monomer (a) Example of conventional method

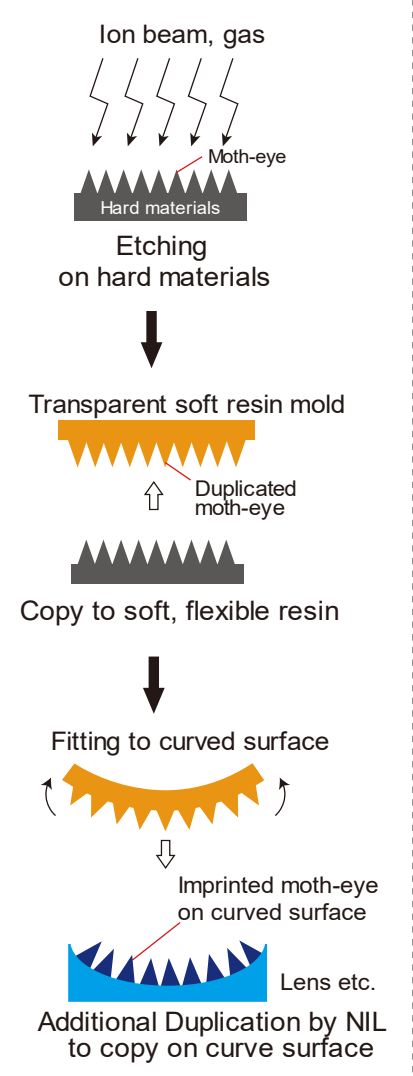

(b) Proposed method

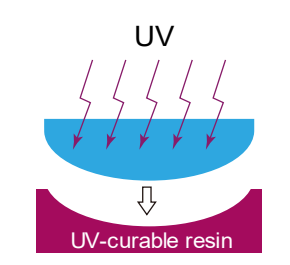

UV imprint

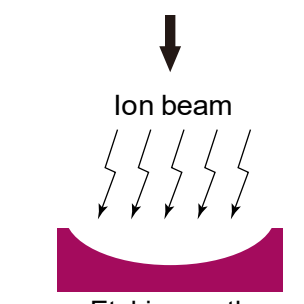

Etching on the UV imprinted polymer lesr

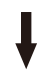

Direct-formed anti-reflector $(500 \mathrm{~nm}-700 \mathrm{~nm})$
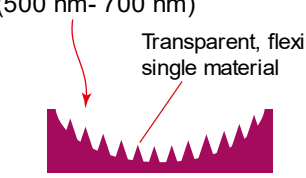

Anti-reflection structure on curved surface
Fig. 1. Comparison of the conventional and proposed moth-eye fabrication methods. (a) Conventional method, which involves etching the moth-eye structure onto hard materials, and then duplicating this to a soft, transparent, and flexible resin replica mold. (b) Proposed method to form moth-eye structures directly onto UV-curable polymers via oxygen ion-beam etching. (2,2-bis(4-glycidyloxyphenyl)propane; Tokyo Chemical Industry Co., Ltd., Tokyo, Japan), and a photo-acid generator (PAG) (CPI-100P; San-Apro Ltd., Tokyo, Japan) as the photopolymerization initiator; the chemical structures are shown in Fig. 2(a) and (b), respectively. PAG (0.01 g) was directly mixed into the monomer $(0.5 \mathrm{~g})$ in a hot-water bath $\left(85^{\circ} \mathrm{C}\right)$ with ultrasonic mixing for 10 minutes. This mixture produced a UV-curable resin by cationic ringopening polymerization of the epoxy functional groups [21, 22].

\subsection{Processes}

Our process produces moth-eye-like structures on a UV-cured polymer surface in two-step process: UV lithography (e.g. UV-NIL) and oxygen ion-beam etching. The fabrication process is schematically shown in Fig. 3a and $3 \mathrm{~b}$ for flat and curved surfaces, respectively.

For the flat surface, the UV-curable resin droplet was first dropped onto a cut $\mathrm{Si}$ wafer substrate $(15 \mathrm{~mm}$ $\times 15 \mathrm{~mm})$ and spin-coated with a rotational speed of 3000 rpm (Fig. 3(a-1)). We note that we coated the Si wafer substrate with hexamethyldisilazane (HMDS) (Tokyo Chemical Industry Co., Ltd., Tokyo, Japan) to improve the adhesion between the Si substrate and the resin layer. The HMDS-coated-substrate was baked on a hot plate at $120^{\circ} \mathrm{C}$ for 20 minutes. After baking, the substrate was washed with acetone. The spincoated resin layer was then cured with a UV lamp (Aicure UP-50; Panasonic Co., Osaka, Japan) with a UV dose and peak wavelength of $60 \mathrm{~mJ} / \mathrm{cm}^{2}$ and 365 nm, respectively (Fig. 3(a-2)). The cured layer was etched using an oxygen ion beam produced by an inductively coupled-plasma (ICP) etching machine (EIS-700; ELIONIX Inc., Tokyo, Japan) with a power and oxygen flow rate of $500 \mathrm{~W}$ and $3.5 \mathrm{sccm}$, respectively (Fig. 3(a-3). The distance from the beam gun to the workpiece was $110 \mathrm{~mm}$. Three different etching times were used $(90,120$, and 150 s). Finally
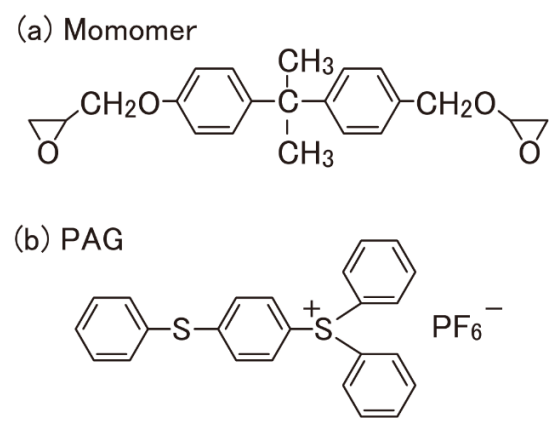

Fig. 2. Materials used for the UV-curable resin: (a) monomer; (b) photopolymerization initiator. 
(a) Flat condition

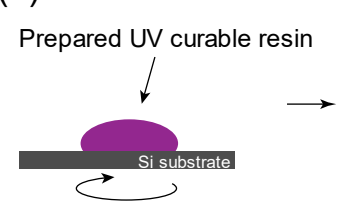

(1) Spin coating

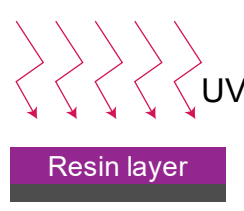

(2) UV curing

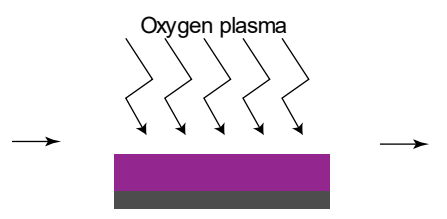

(3) Dry etching by ICP
Moth-eye-like structure

UV polymer moth-eye

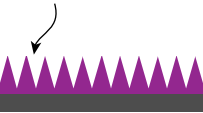

(4) Obtain polymer motheye

(b) Curved condition

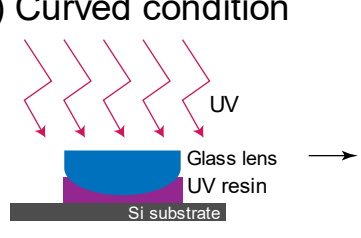

(1) Duplicating an original lens

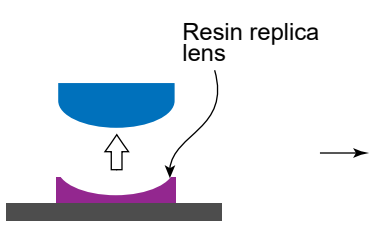

(2) Removing the original lens

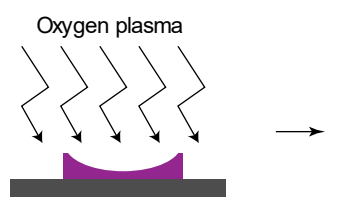

(3) Dry etching by ICP

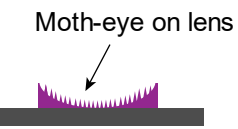

(4) Obtain moth-eye on lens

Fig. 3. Fabrication process of moth-eye structures on a UV-cured polymer using ICP etching. (a) Flat surface: (a-1) spin coating of the resin on a Si substrate; (a-2) UV curing; (a-3) oxygen plasma etching by ICP without masking; and (a-4) the moth-eye structure obtained on the polymer surface. (b) Curved surface: (b-1) duplication of the original lens by UV curing; (b-2) removal of the original lens; (a-3) oxygen plasma etching onto the duplicated concave lens without masking; and (b-4) the moth-eye structure obtained on the surface of the concave lens.

(Fig. 3(a-4)), a layer with moth-eye-like structures was obtained on the flat substrate.

To form the structures on a curved surface, we fabricated a concave lens duplicated from a convex lens mold. As depicted in Fig. 3(b-1), the UV resin was first UV imprinted to form the duplicated lens. The resin lens was duplicated on a Si wafer substrate for easy handling. The UV light was irradiated through the transparent glass lens mold with a UV dose of 60 $\mathrm{mJ} / \mathrm{cm}^{2}$. A release agent was coated of the surface of the lens mold by first dipping the lens into a $1.0 \%$ OPTOOL DSX (DAIKIN Industries, Ltd., Osaka, Japan) solution for 3 hours and baking on a hot plate at $100{ }^{\circ} \mathrm{C}$ for 15 minutes. After baking, the lens mold was washed in NOVEC7300 (3M Company, Minnesota, USA) to remove contamination. Owing to the release coating, the lens mold was released smoothly from the UV cured resin, and a concave resin lens was obtained. Next, the duplicated concave resin lens was etched with the oxygen ion beam under the same etching conditions as described above (Fig. 3(b$3)$ ). Finally, the moth-eye-like structure was obtained directly on the concave resin lens (Fig. 3(b-4)). Each sample was observed using a scanning electron microscope (SEM; ERA8800FE, ELIONIX Inc., Tokyo, Japan) and its reflectance was measured using a spectrophotometer (Solidspec-3700; Shimadzu Corp., Kyoto, Japan).

\section{Results and discussion}

\subsection{Flat surface}

An SEM image of the etched layer on a flatpolished Si substrate for an etching time of $120 \mathrm{~s}$ is shown in Fig. 4. The moth-eye structure was formed on the UV-cured polymer layer, which had a thickness of $44.7 \mu \mathrm{m}$, as seen in Fig. 4(a). Pillar structures, as shown in Fig. 4(b), covered the surface of the $20 \mathrm{~mm} \times 20 \mathrm{~mm}$ polymer layer. The average pillar height, diameter, and pitch were 700 , 300 , and $100 \mathrm{~nm}$, respectively. Thus, this single etching process can produce high-aspect-ratio moth-eye-like nanostructures. The shape and height of the structures were almost same as those formed on glassy carbon.

We measured the reflectance from the flat surface. Figure 5 shows the reflectance ratio before and after etching for etching times of 90, 120, and $150 \mathrm{~s}$. Figure 5(a) and (b) show the reflectance for initial layer thicknesses (i.e. before etching) of 26 (condition A) and $92 \mu \mathrm{m}$ (condition B), respectively. The reflectance decreased with etching time for both conditions, reaching $7 \%$ after $150 \mathrm{~s}$. Thus, the oxygen ion beam effectively produced antireflective structures onto the UVcured polymer layer. Figure 5(c) shows the relationship between the etching time and average reflectance in conditions A and B. For each etching time, the decrease in reflectance was similar for both conditions. On the basis of these results, we suggest that the height of the moth-eyelike structure was independent of the initial thickness. Therefore, it is possible to add an antireflection structure to films of any thickness.

\subsection{Curved surface}

Next, we fabricated moth-eye-like structures on concave and convex polymer lenses, which were 

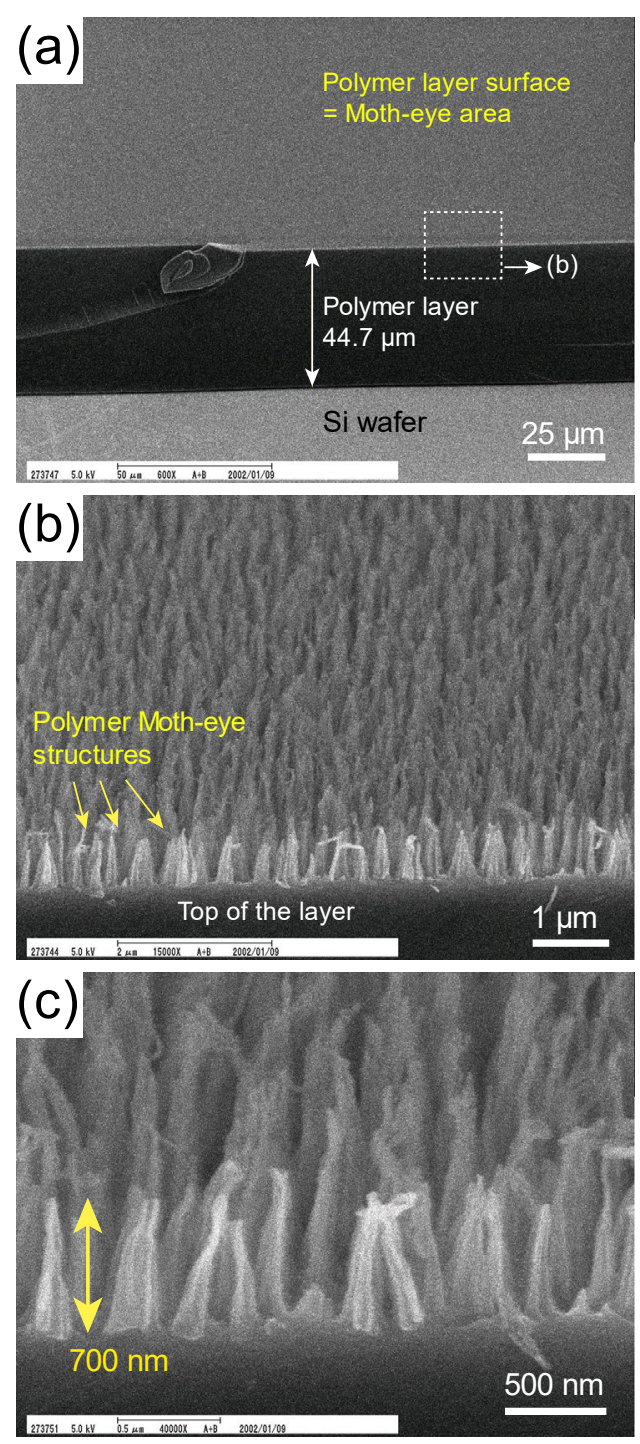

Fig. 4. Cross-sectional SEM images of the moth-eyelike nanostructure on a flat surface. (a) Etched UVcured polymer layer. (b) Pillar structures on the polymer layer. (c) Higher-magnification image of the pillars, from which the average height and pitch were measured to be 200 and $700 \mathrm{~nm}$, respectively.

duplicated by UV imprinting from original glass lenses, as schematically shown in Fig. 3(b). Figure $6 \mathrm{a}$ and $6 \mathrm{~b}$ show SEM images of the etched concave and convex lenses, respectively. In this demonstration, the concave lens was shaped on a substrate and the convex lens was shaped as a stand-alone-lens. In each sample, bumpy structures were formed over the entire surface. The pitch of the structure was wider than that created on the flat surface. The height of the nanostructures on both the concave and convex lenses was $700 \mathrm{~nm}$. However, the width of the nanostructures was smaller on the convex lens than on the concave lens. In summary, moth-eye- like structures formed on the UV-imprinted replica lenses.

The reflectance was measured for both etched lenses with the method described above for the flat surface. Figure $7 \mathrm{a}$ and $7 \mathrm{~b}$ show the reflectance of the concave and convex lenses, respectively; the reflectance from the non-etched lenses is shown for comparison. For both lenses, the reflectance decreased after etching, although the extent of the reduction was much greater for the concave lens. This demonstrates that AR nanostructures could be etched in a single step using the oxygen ion beam. On the basis of these results, we expect that our method will improve future manufacturing processes for AR lenses and their molds.
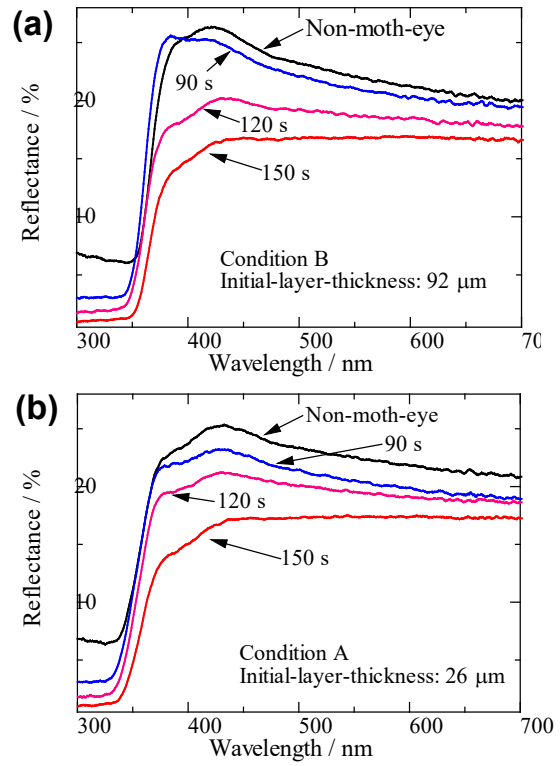

(c)

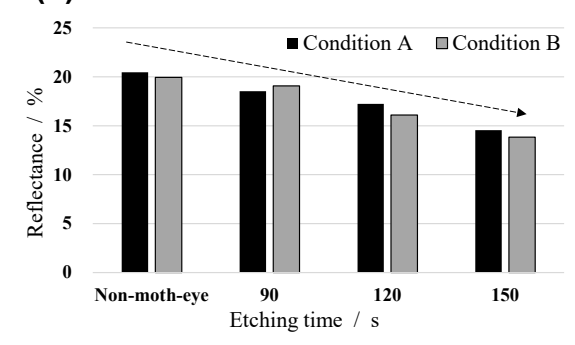

Fig. 5. Reflectance of the etched surfaces for etching times of 90,120 , and $150 \mathrm{~s}$ for initial thicknesses of (a) $26 \mu \mathrm{m}$ (condition A) and (b) $92 \mu \mathrm{m}$ (condition B). (c) Comparison of the reflectance for conditions $\mathrm{A}$ and $\mathrm{B}$ for each etching time.

\subsection{Etching onto a microlens array}

Finally, we formed a moth-eye-like structure on a UV-imprinted lens array. The process involved first UV-imprinting a lens array on a primer coated $\mathrm{Si}$ wafer with a UV dose of $60 \mathrm{~mJ} / \mathrm{cm}^{2}$. The convex 

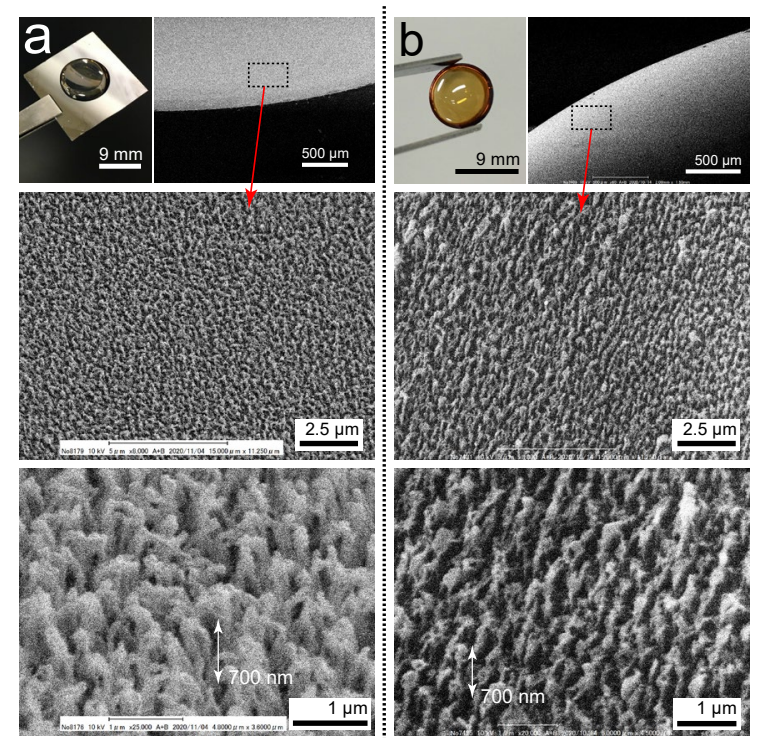

Fig. 6. SEM images of the moth-eye-like nanostructures etched on (a) concave and (b) convex polymer lenses shaped by UV imprinting.

(a) Curve condition: Concave lens
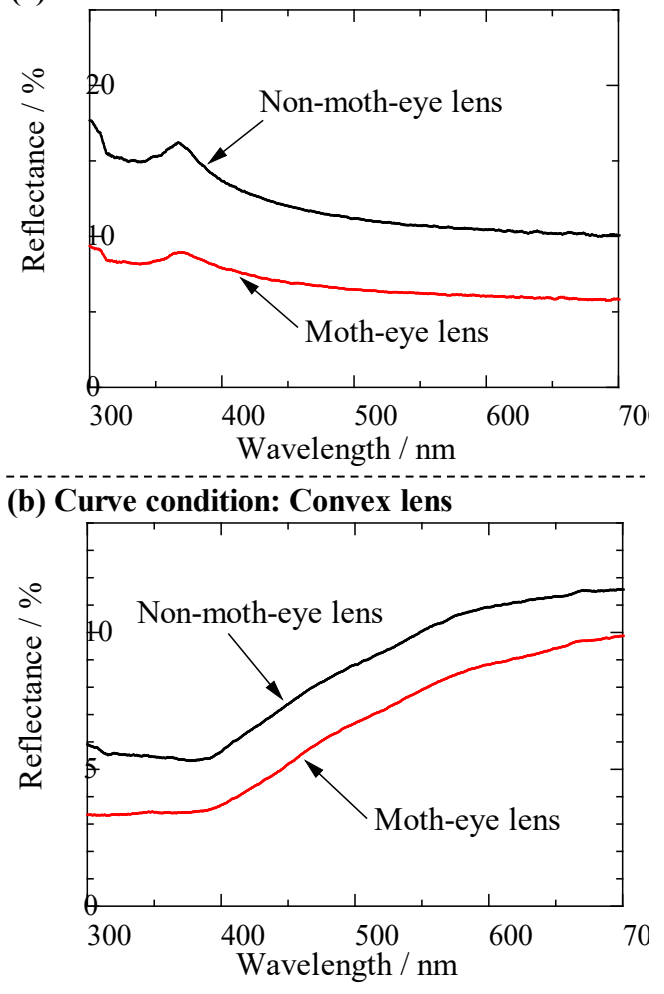

Fig. 7. Comparison of reflectance of the moth-eyeetched and non-etched (a) concave and (b) convex lenses.

lens array was imprinted from a glass convex master mold. As shown in Fig. 8, the moth-eye-like nanostructure formed over the entire lens array with
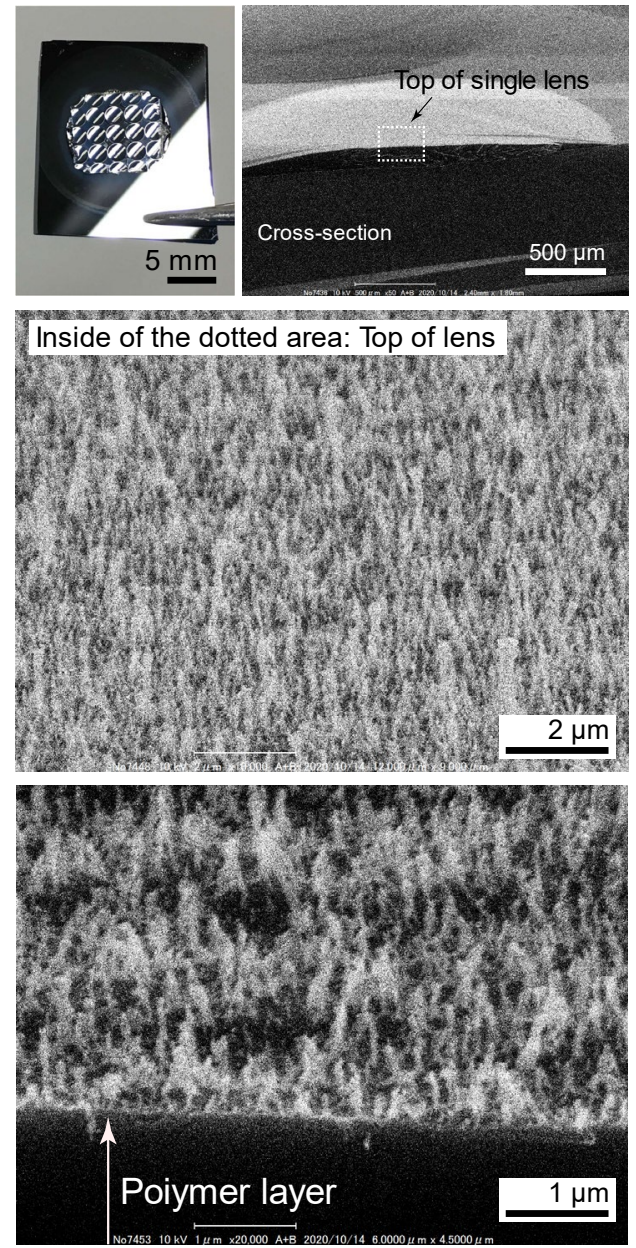

Fig. 8. Photograph and SEM images of the moth-eyelike nanostructures formed on a UV-imprinted convex microlens array.

an average pillar height of approximately $700 \mathrm{~nm}$. Thus, our method can even create moth-eye-like structures by etching on regular patterns formed by UV-NIL. We expect that this method will be used to fabricate moth-eye structures on microscale patterns using NIL

\section{Conclusion}

In this paper, we proposed a simple method to fabricate moth-eye-like AR nanostructures via oxygen ion-beam etching directly onto a UVcurable polymer. The moth-eye-like nanostructures had a high aspect ratio and an average height of 700 $\mathrm{nm}$. For both flat and curved lenses, the reflectance was substantially reduced after etching, demonstrating the potential of these surfaces for AR applications. We also formed moth-eye-like structures on a UV-imprinted lens array. The method can make moth-eye-like nanostructures on patterns formed by the UV-NIL process. In summary, our method allows the low-cost 
production of polymer lenses with AR functions and their NIL molds.

\section{References}

1. S. M. Kang, S. Jang, J. K. Lee, J. Yoon, D. E. Yoo, J. W. Lee, M. Choi, and N. G. Park, Small, 12 (2016) 2443.

2. G. Tan, J. H. Lee, Y. H. Lan, M. K. Wei, L. H. Peng, I. C. Cheng, and S. T. Wu, Optica, 4 (2017) 2334.

3. Y. Uozu, J. Photopolym. Sci. Technol., 32 (2019) 291.

4. S. S. Oha, C. G. Choi, and Y. S. Kim, Microelectron. Eng., 87 (2010) 2328.

5. S. Xie, X. Wan, B. Yang, W. Zhang, X. Wei, and S. Zhuang, Nanomaterials, 9 (2019) 747.

6. P. B. Clapham and M. C. Hutley, Nature, 244 (1973) 281.

7. T. Yano, H. Sugawara, and J. Taniguchi, Microelectron. Eng., 9 (2020) 100077.

8. I. Mano, T. Uchida, and J. Taniguchi, Microelectron. Eng., 191 (2018) 97.

9. H. Eto, S. Hiwasa, and J. Taniguchi, J. Photopolym. Sci. Technol., 30 (2017) 533.

10. T. Yanagishita, T. Kondo, and H. Masuda, $J$. Vac. Sci. Technol. B, 36 (2018) 031802.

11. S. Ji, J. Park and H. Lim, Nanoscale, 4 (2012) 4603.

12. S. Ji, K. Song, T. B. Nguyen, N. Kim, and H. Lim, ACS Appl. Mater. Interfaces, 5 (2013) 10731.
13. M. R. Lotz, C. R. Petersen, C. Markos, O. Bang, M. H. Jakobsen, and R. Taboryski, Optica, 5 (2018) 557.

14. T. Senn, O. Kutz, C. Weniger, J. Li, M. Schoengen, H. Lochel, J. Wolf, P. Gottert, and B. Lochel, J. Vac. Sci. Technol. B, 29 (2011) 061601.

15. J. W. Leem, X. Y. Guan, M. Choi, and J. S. Yu, Sol. Energ. Mat. Sol. C, 134 (2015) 45.

16. S. Ju, J. Y. Choi, D. Chae, H. Lim, H. Kang, and H. Lee, Nanotechnology, 31 (2020) 505301.

17. J. S. Choi, J. H. An, J. K. Lee, J. Y. Lee, and S. M. Kang, Polymers, 12 (2020) 296.

18. C. Zhang, P. Yi, L. Peng, X. Lai, and J. Ni, IEEE Trans. Nanobioscience, 14 (2015) 1127.

19. Q. Chen, G. Hubbard, P. A. Shields, C. Liu, D. W. E. Allsopp, W. N. Wang, and S. Abbott, Appl. Phys. Lett., 94 (2009) 263118.

20. I. N. Baena, A. J. Martín, J. J. Hernández, J. R. C. Smirnov, F. Viela, M. A. Monclús, M. R. Osorio, J. M. M. Aldareguia, and I. Rodríguez, Nanoscale, 10 (2018) 15496.

21. X. Ning and H. Ishida, Polym. Sci. A Polym. Chem. 32 (1994) 1121.

22. G. Liu, X. Zhu, B. Xu, X. Qian, G. Song, and J. Nie, Appl. Polym. Sci. 130 (2013) 3698. 